\title{
Jab1 promotes gastric cancer tumorigenesis via non-ubiquitin proteasomal degradation of p14ARF
}

\author{
Lin Wang $^{1,2} \cdot$ Wen-Qi Du ${ }^{1,3} \cdot$ Min Xie $^{1} \cdot$ Man-Ru Liu ${ }^{1} \cdot$ Fu-Chun Huo ${ }^{1} \cdot$ Jing Yang $^{4} \cdot$ Dong-Sheng Pei $^{1}$
}

Received: 3 April 2020 / Accepted: 16 May 2020 / Published online: 26 May 2020

(c) The International Gastric Cancer Association and The Japanese Gastric Cancer Association 2020

\begin{abstract}
Background Jab1 has been reported to regulate various proteins in signal transduction pathways and be implicated in carcinogenesis or tumor progression. However, the precise role and molecular mechanism of Jab1 in gastric tumorigenesis have not yet been fully elucidated.

Methods Jab1 staining in gastric cancer tissues and paired non-cancerous tissues was measured using tissue microarray (TMA) technology. The impact of Jab1 on tumor growth in vivo was analyzed using xenotransplantation experiments in $\mathrm{Balb} / \mathrm{c}$ mice. The expression of Jab1 and p14ARF in gastric cancer cells was analyzed by western blot and confocal immunofluorescence. CCK-8 and cell cycle experiment were used to evaluate the cell proliferation. Ubiquitination assay was performed to validate whether ubiquitination is involved in Jab1-mediated p14ARF degradation.

Results The expression level of protein p14ARF was inversely correlated with the protein level of Jab1. Then, we investigated the mechanism that how Jab1 induced p14ARF depletion. Mechanistic studies showed that Jab1 induced ubiquitinindependent proteasomal p14ARF degradation in gastric cancer cells. Our data demonstrated that Jab1 protein was a vital upstream negative modulation factor of p14ARF, and Jab1 could promote cell proliferation and tumor growth via inhibiting the expression of p14ARF in vivo and in vitro. Moreover, silencing Jab1 protein expression declined tumor growth and further increased the apoptosis rate of gastric cancer cells. In further studies of gastric cancer specimens, we found the increased level of Jab1 protein shortened the overall survival.

Conclusion Jab1 is upstream of p14ARF and promote gastric cancer cell proliferation in vitro and in vivo. Furthermore, Jab1 decreased the expression of p14ARF though ubiquitination independent proteasomal degradation. Therefore, the connection of Jab1 and p14ARF may provide new methods for the treatment of gastric cancer.
\end{abstract}

Keywords Jab1 · p14ARF · Cell proliferation · Apoptosis · Gastric cancer

Lin Wang and Wen-Qi Du contributed equally to this paper.

Electronic supplementary material The online version of this article (https://doi.org/10.1007/s10120-020-01087-z) contains supplementary material, which is available to authorized users.

Dong-Sheng Pei

dspei@xzhmu.edu.cn

1 Department of Pathology, Xuzhou Medical University, 209 Tong-shan Road, Xuzhou 221004, Jiangsu, China

2 Department of Respiratory Medicine, Second Affiliated Hospital of Xuzhou Medical University, Xuzhou 221004, China

3 Department of Human Anatomy, Xuzhou Medical University, Xuzhou 221004, China

4 Department of Oncology, Xuzhou Hospital Affiliated to Nanjing University of Chinese Medicine, Xuzhou 221003, China

$\begin{array}{ll}\text { Abbreviations } \\ \text { GC } & \text { Gastric cancer } \\ \text { Jab1 } & \text { C-Jun activation domain-binding protein 1 } \\ \text { CSN } & \text { COP9 signalosome } \\ \text { OS } & \text { Overall survival } \\ \text { Co-IP } & \text { Co-immunoprecipitation assay } \\ \text { IHC } & \text { Immunohistochemical } \\ \text { CHX } & \text { Cycloheximide } \\ \text { BCA } & \text { Bicinchoninic acid } \\ \text { WCL } & \text { Whole cell lysate } \\ \text { ODC } & \text { Ornithine decarboxylase }\end{array}$




\section{Introduction}

Gastric cancer (GC) is the fourth highest incidence of cancers and the third most lethal malignancy worldwide [1]. Most patients with GC comprise metastasis at the time of diagnosis and lose the chance of radical operation due to the no symptoms or misdiagnosis in its early stages [2]. Despite standard treatment, many GC patients still have recurrence and metastasis. Clinical outcomes of GC patients are still suboptimal, and its 5-year survival rate is less than $30 \%$ [3]. Therefore, it is urgent to explore early diagnosis mark and therapy target for GC.

p14ARF is a tumor suppressor gene that specializes in regulating the cell cycle mainly through $\mathrm{p} 53$-dependent and $\mathrm{p} 53$-independent pathways to inhibit tumor occurrence [4-6]. The INK4a-ARF gene produces p14ARF as an alternative reading frame (ARF: p14ARF in humans and p19ARF in mice) $[4,7]$. INK4a-ARF is a multi-coding gene locus containing two promoters in mammalian cells, INK4a-ARF also encodes the p16-INK4a protein $[8,9]$. p14ARF and p16-INK4a sharing exons 2 and exons 3 present different reading frames, the amino acid sequence of both is completely non-homologous and molecular mechanisms of p14ARF and p16-INK4a in tumor inhibition are actually independent $[8,10]$. Many studies have validated that $\mathrm{p} 14 \mathrm{ARF}$ is inactivated in many primary tumors, although its inactivation mechanism varies. It has been confirmed that p14ARF shows ectopic expression in lung cancer, liver cancer and intestinal tumors and works as an antioncogene $[8,11,12]$. However, the mechanism underlying p14ARF depletion in GC has not yet elucidated.

c-Jun activation domain-binding protein 1 (Jab1), also referred to as the fifth subunit (CSN5) of COP9 signalosome (CSN) complex $[13,14]$, is a protein screened from the cDNA library of human lymphocytes [15]. Deng et al. first discovered the CSN complex and considered it as an essential regulator of plant photomorphogenesis in Arabidopsis [16]. Amplification and overexpression of Jab1 are found in multiple malignant tumors, and it could be proposed as a tumor marker. Many studies have already confirmed that Jab1 dysregulation contributes to tumorigenesis by interacting with or functionally regulating the stability and activity of several pivotal proteins, including the cyclin-dependent kinase inhibitors p57 [17], p27 [18], HIF-1 $\alpha$ [19], and others $[20,21]$. In our preliminary experiments, the expression of Jab1 protein in GC was relatively increased and the p14ARF expression was reduced. Therefore, we hypothesized that Jab1 functions as a vital negative regulator of p14ARF as well as to promote malignant biological function of GC cells, and negatively correlates with the prognosis of GC.

In this study, we confirmed that high expression of Jab1 was associated with a shortened overall survival (OS) in
Fig. 1 Expression patterns of Jab1 in tissues of gastric cancer patients and prognostic significance. a, b Jab1 protein expression was shown in 20 pairs of human gastric cancer tissues and para-cancer. Western Blots (WB) analysis of Jab1 in four representative paired samples of para-tumor tissue $(\mathrm{P})$ and gastric cancer tissue $(\mathrm{T})$ are shown. $\beta$-actin was used as a control for protein load and integrity. The right image showed quantification of the Jab1 protein expression (data represent the mean \pm SEM, $P<0.01)$. c Protein levels of Jab1 in para-tumor and malignant tumor tissues in gastric cancer patients. In situ expression of Jab1 was detected by hematoxylin and eosin (H\&E) staining and immunohistochemical (IHC) analysis as described in "Materials and methods". After identification by H\&E (the upper), the representative cases with high (case 3) and low (case 6) expression of Jab1 protein were analyzed by IHC staining (the bottom). The original magnification is $\times 100$ and $\times 200$, respectively. d Relationship between Jab1 expression patterns and overall survival rates (OS) in 90 gastric cancer patients. Kaplan-Meier curves show that Jab1 amplification was associated with a poorer prognosis and a shorter OS $(P=0.0029)$. $* * P<0.01$

90 cases of patients with GC, and increased Jab1 facilitated cell proliferation and cycle progression of GC. We measured and confirmed the inverse correlations between Jab1 and p14ARF proteins both in vivo and in vitro. Coimmunoprecipitation assay (Co-IP) showed a physical interaction between Jab1 and p14ARF. We also found that Jab1-mediated degradation of p14ARF was not via 26S-proteasome pathway, but rather through the ubiquitinindependent manner involved PA200, an activator of $20 \mathrm{~S}$ proteasome $[22,23]$. Our results suggest that Jab1 mediated p14ARF degradation may affect tumorigenesis and thus be a promising therapeutic target for human GC.

\section{Materials and methods}

\section{Cell culture and transfection}

Human GC cell lines MKN45 and AGS were obtained from the Institute of Biochemistry and Cell Biology, Chinese Academy of Sciences (Shanghai, China) and cultured in RPMI-1640 medium (Grand Island, NY, USA) containing $10 \%$ fetal bovine serum (Invitrogen, Carlsbad, CA, USA) and penicillin-streptomycin sulfate. Cells were incubated at $37{ }^{\circ} \mathrm{C}$ and in an atmosphere of $5 \% \mathrm{CO}_{2}$. For the interfering assay, Jab1 siRNA (Si-Ctrl, sense: 5'-UUCUCCGAA CGUGUCACGUTT -3', Si-Jab1, sense: 5'-CCAGACUAU UCCACUUAAUTT-3'), as previously described [24], was obtained from GenePharma (Shanghai, China). In addition, the oligonucleotides of Si-Jab1 and control-siRNA ( $\mathrm{Si}$ control) were transfected into human GC cells by siLentFect Lipid Reagent (Bio-Rad, Hercules, IN, USA) and incubated for $48 \mathrm{~h}$. Transfection of MKN45 and AGS cells with Jab1 plasmids, p14ARF plasmids, and control plasmids (GenePharma, Shanghai, China) by X-tremeGENE HP 
a
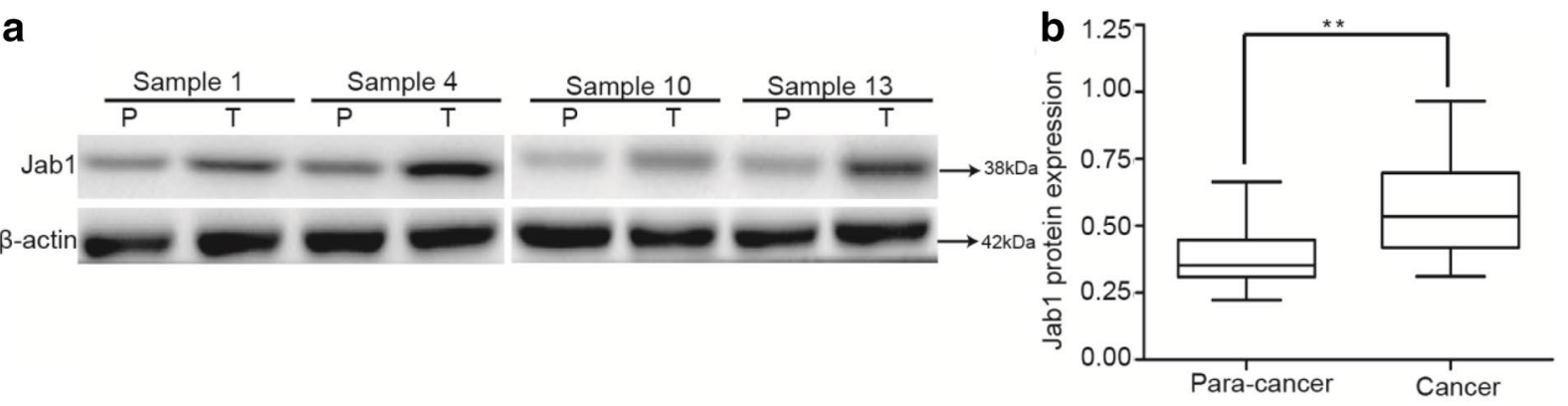

C

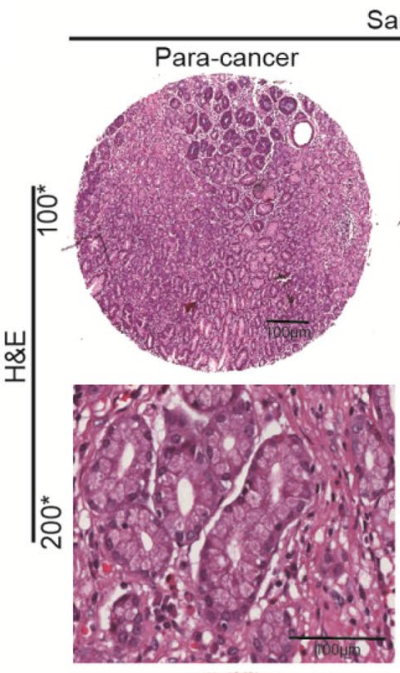

Sample 3
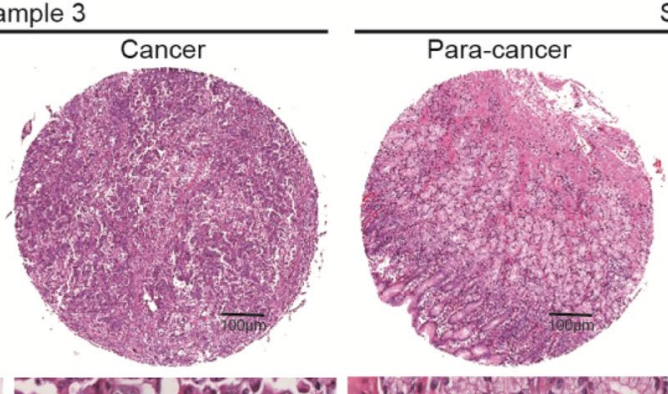

Sample 6

Cancer
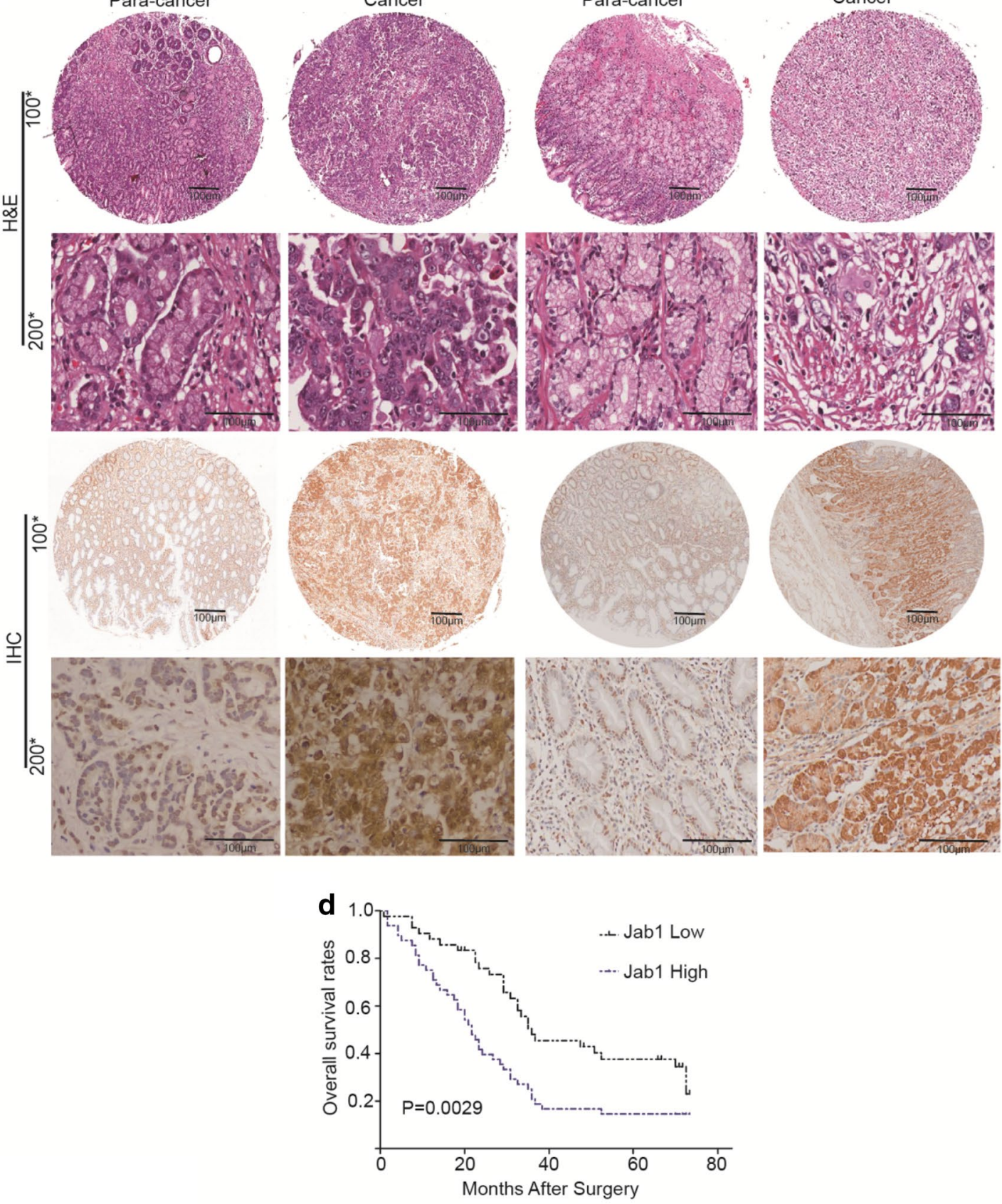
DNA Transfection Reagent (Roche, Indianapolis, IN, USA). Lentiviral vectors containing Jab1 plasmids were purchased from GenePharma, MKN45 cells stably transfected with the lentivirus and screening for 2-4 weeks.

\section{Human tumor samples}

20 pairs of primary gastric tumors and adjacent tissues were obtained from the Affiliated Hospital of Xuzhou Medical University. Collection and usage of all patient materials and information were conducted according the institutional guidelines and the Declaration of Helsinki Principles. Primary gastric tumor samples were obtained from patients who had undergone operations to treat GC. Informed consent was obtained for use of these pathologic samples for research.

\section{Tissue microarray and IHC staining}

We obtained 90 pairs of gastric cancer and para-cancer specimens from surgical resections that were performed at the Affiliated Hospital of Xuzhou Medical University between December 1th, 2010 and April 30th, 2011. And the tissue microarray was constructed. The slides were incubated with anti-Jab1 antibody. Immunohistochemical (IHC) staining score was obtained by immunoreactive scores (IRS), which is calculated by multiplying the score of intensity and the score of extent. Staining intensity was scored $0-3(0=$ negative; $1=$ weak; $2=$ moderate; $3=$ strong). The extent of positive stained cells was also scored into 4 categories: 1 (0\%-25\%), 2 (26\%-50\%), $3(51 \%-75 \%)$, and 4 (76\%-100\%). Based on the IRS, the staining pattern was defined as low (IR: 0-6) and high (IRS 8-12). Informed consent has been obtained from each patient. This study was approved by the Review Board of the Affiliated Hospital of Xuzhou Medical University.

\section{Functional analysis of cell biology}

For the cell viability assay, after being transfected with Jab1 plasmids or Si-Jab1, MKN45 and AGS cells $\left(5 \times 10^{3}\right)$ were incubated in 96-well plates for 24, 48, 72, and $96 \mathrm{~h}$, Cell Counting Kit-8 (CCK-8, Beyotime Biotechnology, Beijing, China) was be applied to evaluate the ability of cell proliferation. The growth and apoptosis of cells and the change of cell cycle were measured using Annexin V-FITC Apoptosis Detection Kit (BD Biosciences, San Jose, CA, USA) and PI/ RNase Staining Buffer (BD Biosciences, USA) respectively, as previously described [24]. Immunofluorescence assays were performed to elucidate the expression and localization of Jab1 and p14ARF in GC cells. MKN45 cells were transfected with Jab1 and/or p14ARF plasmids for $24 \mathrm{~h}$, and the following detection method used was consistent with the previous methods [25]. Finally, the cells were incubated with Alexa Fluor 488-conjugated anti-mouse and/or TRITCconjugated anti-rabbit antibodies and visualized using Laser Scanning Confocal Microscope.

\section{Western blot analysis and Co-IP assays}

Following different treatments of cells, such as transfection and incubation with MG132 $(50 \mu \mathrm{g} / \mathrm{mL}$, Selleck Biotechnology, Houston, TX, USA), Cycloheximide (100 $\mu \mathrm{g} / \mathrm{ml}$, CHX, Sigma, St Louis, MO, USA), Hydroxychloroquine ( $30 \mu \mathrm{M}$, Selleck chemicals, Houston, TX, USA), Pepstatin A (70 $\mu \mathrm{M}$, Selleck chemicals, Houston, TX, USA) and 3-Methyladenine (10 mM, 3-MA, Selleck chemicals, Houston, TX, USA), cells were harvested and the lysates were detected using a bicinchoninic acid (BCA) Kit (Beyotime Biotechnology). Protein samples were boiled in $5 \times$ loading buffer, electrophoresed by $15 \%$ SDS-PAGE and transferred onto nitrocellulose membranes. After blocking for $2 \mathrm{~h}$ in $5 \%$ skim milk, membranes were incubated overnight at $4{ }^{\circ} \mathrm{C}$ with the designated antibodies: Jab1, $\beta$-actin, (Santa Cruz Biotechnology, Santa Cruz, CA, USA); p14ARF (Abcam Cambridge,

Table 1 Clinical characteristics of 90 gastric cancer patients and Jab1 expression

\begin{tabular}{|c|c|c|c|}
\hline & \multirow{2}{*}{$\begin{array}{l}\text { Low expression } \\
\text { Of Jab1 }(n=35)\end{array}$} & \multicolumn{2}{|c|}{ High expression } \\
\hline & & $\begin{array}{l}\text { Of Jab1 } \\
(n=55)\end{array}$ & $P$ value* \\
\hline \multicolumn{4}{|l|}{ Sex } \\
\hline Female & 9 & 11 & 0.606 \\
\hline Male & 26 & 44 & \\
\hline \multicolumn{4}{|c|}{ Age (years) } \\
\hline$\leq 50$ & 5 & 4 & 0.302 \\
\hline$>50$ & 30 & 51 & \\
\hline \multicolumn{4}{|c|}{ Tumor size $(\mathrm{cm})$} \\
\hline$\leq 5$ & 21 & 14 & 0.002 \\
\hline$>5$ & 14 & 41 & \\
\hline \multicolumn{4}{|c|}{ Tumor stage } \\
\hline I-II & 20 & 19 & 0.049 \\
\hline III-IV & 15 & 36 & \\
\hline \multicolumn{4}{|l|}{ pT status } \\
\hline $\mathrm{pT}_{1-2}$ & 18 & 12 & 0.002 \\
\hline $\mathrm{pT}_{3}$ & 11 & 15 & \\
\hline $\mathrm{pT}_{4}$ & 6 & 28 & \\
\hline \multicolumn{4}{|l|}{ pN status } \\
\hline $\mathrm{pN}_{0}$ & 20 & 15 & 0.012 \\
\hline $\mathrm{pN}_{1}$ & 9 & 18 & \\
\hline $\mathrm{pN}_{2-3}$ & 6 & 22 & \\
\hline
\end{tabular}

$P<0.05$ was considered statistically significant. Pearson's chi-square test was used 
MA, USA); p16INK4a (Ruiying Biological, Suzhou, China). Membranes were then washed and incubated with secondary antibodies. Visualized images were obtained by using a Tanon detector (Shanghai, China) and analyzed with Image J software. Briefly, the difference between Co-IP and western blot detection is that before running electrophoresis, the whole cell lysate (WCL) was incubated overnight at $4{ }^{\circ} \mathrm{C}$ with the detection antibodies: PA200, Myc (Abcam) and Flag (Sigma). Subsequently, $30 \mu$ of protein A/G beads (Santa Cruz Biotechnology) were added, and the samples

a

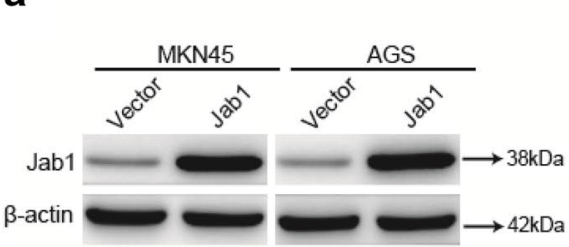

C

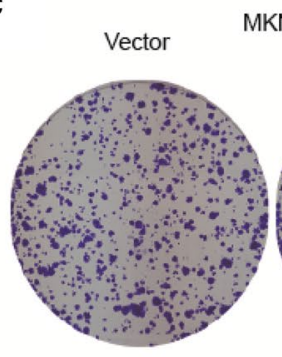

MKN45

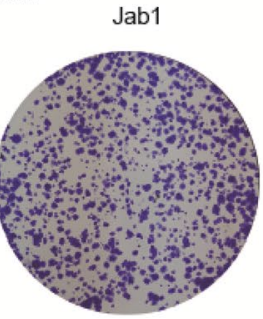

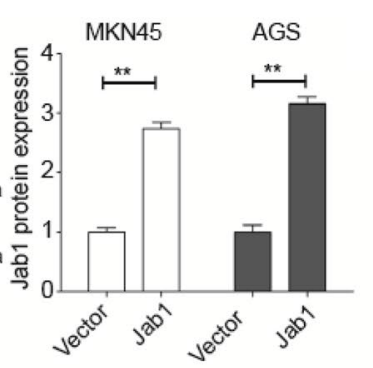

AGS
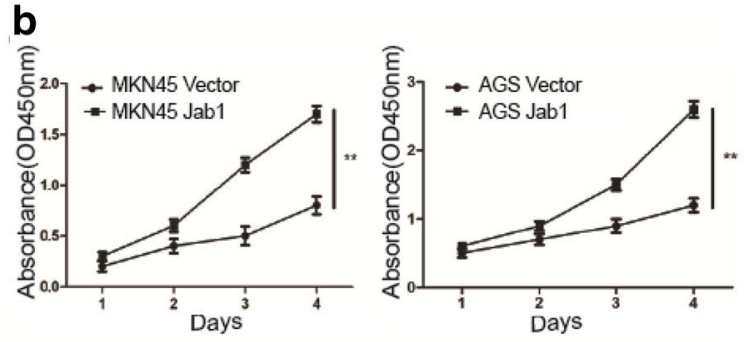

d
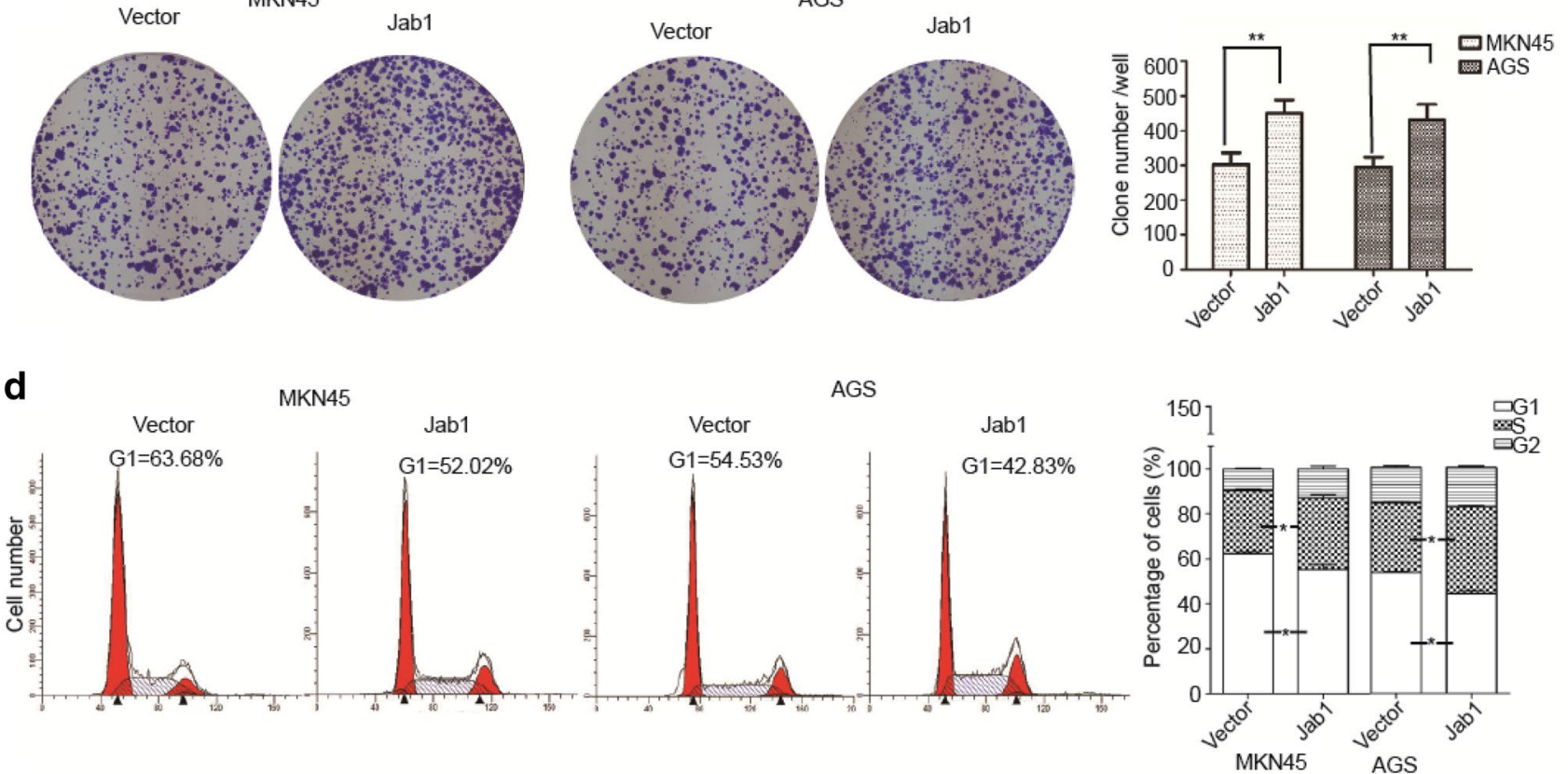

e
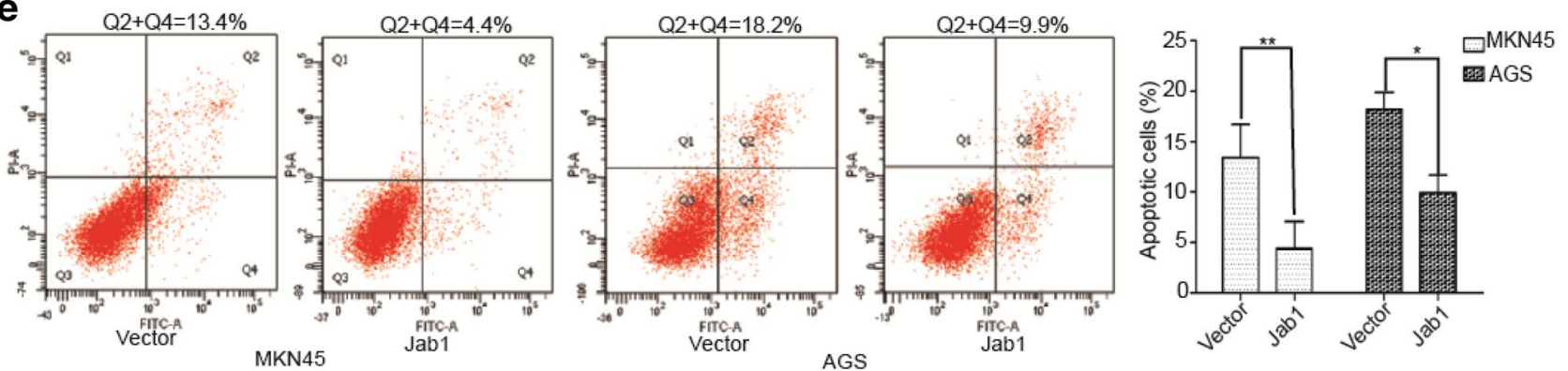

Fig. 2 High level expression of Jab1 is positively correlated with the proliferation of gastric cancer cells. a WB analyses were used to detect the expression efficiency of Jab1 and the level of p14ARF protein when Jab1 gene vector was transfected into two kinds of gastric cancer cells. Quantification of the Jab1 expression (right). b Overexpression of Jab1 by transfected cells with Jab1 vector for the indicated times can increase relative viable cells in a time-dependent manner (24, 48, 72, and $96 \mathrm{~h}$ ), and the number of viable cells was assessed by a CCK- 8 assay. c Clonality in gastric cancer cells (500 cells) treated with the ectopic Jab1 vector or a control vector was confirmed. The quantification of the colonies counting is shown (right). d Flow cytometric (FCM) analysis of the cell cycle following Jab1 overexpression in two kinds of human gastric cancer cells. Right, the proportion of cells in G1 phase is shown. e Apoptosis in cells with forced expression of Jab1 was investigated by FCM. The percentages of apoptotic cells are shown (right). The data are the means \pm standard deviations (SD) of the three independent experiments. $* P<0.05$, $* * P<0.01$ 
a

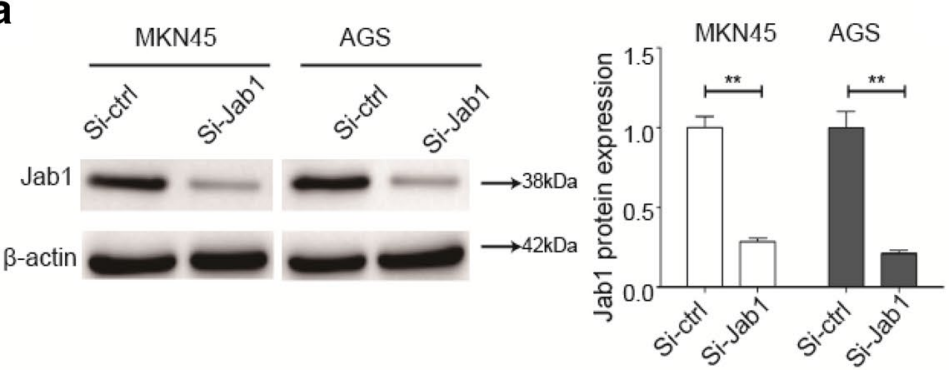

b
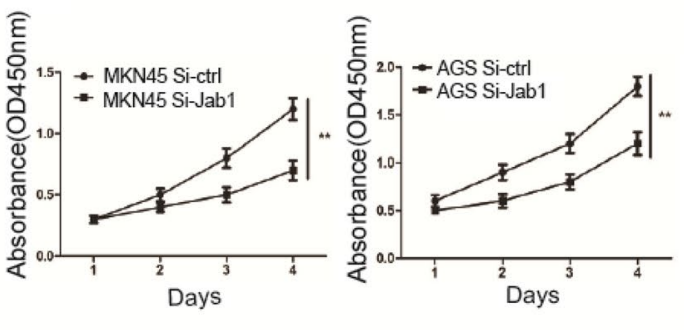

C

MKN45

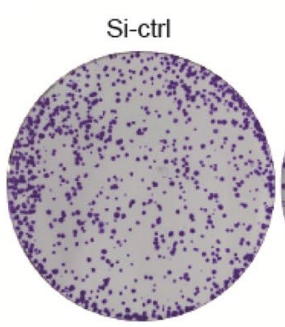

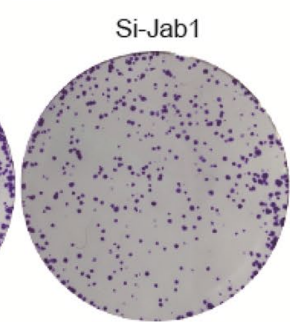
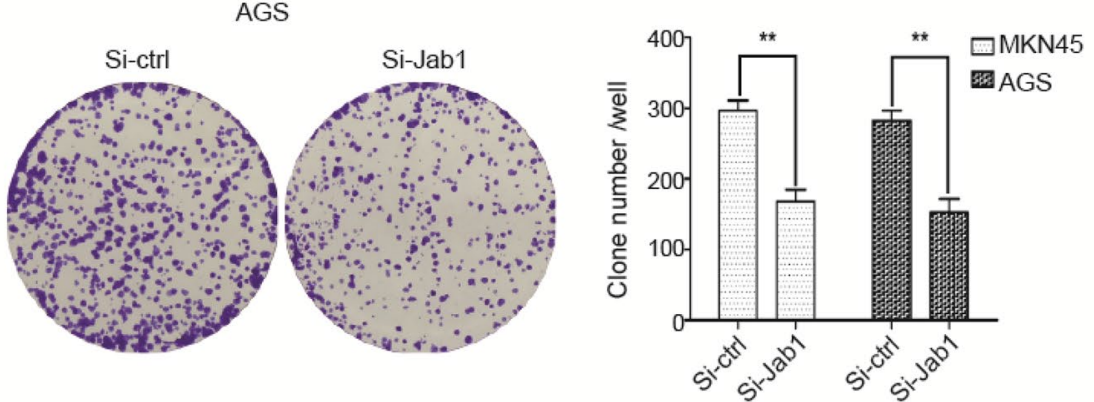

d

MKN45
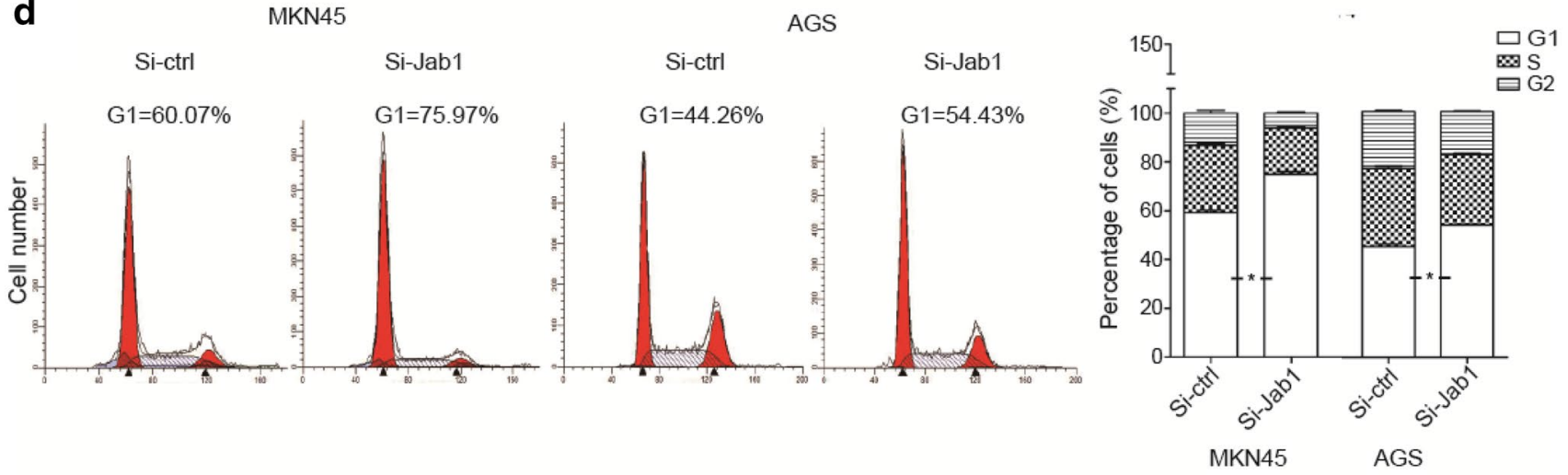

e

$\mathrm{Q} 2+\mathrm{Q} 4=8 \%$

$\mathrm{Q} 2+\mathrm{Q} 4=23 \%$
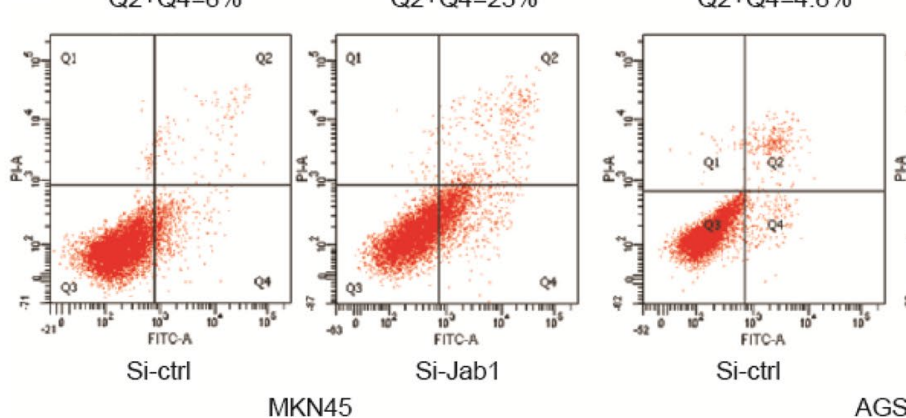

$\mathrm{Q} 2+\mathrm{Q} 4=10.8 \%$
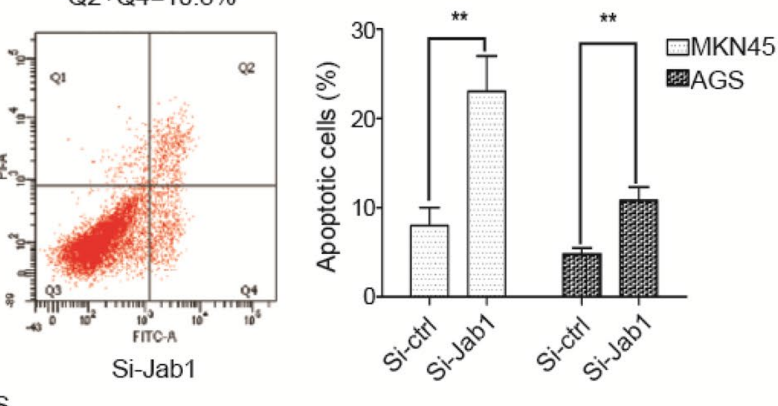
4Fig. 3 Jab1 depletion can mediate the inhibitory function of cell growth and negatively regulates p14ARF protein. a Treatment of Jab1-siRNA (Si-JAB1) suppressed the level of Jab1 expression. Quantification of the Jab1 expression (right). b Cell proliferation retardation in gastric cancer cells with ablation of Jab1 expression was determined by CCK- 8 assay. $\mathbf{c}$ The results of colony formation assays (500 cells) in two human gastric cancer cells transfected with $\mathrm{Si}-\mathrm{Jab} 1$ or Si-control, and the right data are the quantification of relative colonies counting. d Jab1-siRNA treated in MKN45 and AGS cells suppressed the progress of cell cycle. The detection of cell cycle was assessed by FCM and the each group ratio of cells in G1 phase is shown (right). e FCM analysis was used to evaluate the percentages of apoptotic cells when two kinds of gastric cancer cells were interfered with Jab1-siRNA or not. The imaging paraphrase was placed as described in the Materials and Methods. Three independent experiments were performed. $* P<0.05, * * P<0.01$

were mixed by rotation for $4 \mathrm{~h}$. Finally, the supernatant was removed by centrifugation and the samples were detected by $15 \%$ SDS-PAGE electrophoresis.

\section{Ubiquitination assay}

Ubiquitination assay was performed under denaturation conditions using $\mathrm{Ni}^{2+}$-nitrilotriacetic acid beads, as other study groups previously described [26]. The diluted lysate was immunoprecipitated with the anti-p14ARF antibody (Abcam Cambridge) and probed with anti-ub antibody (Santa Cruz Biotechnology).

\section{In vivo tumorigenicity assays}

Following transfection with the lentiviral vectors that contained Jab1 plasmids, MKN45 cells $\left(1 \times 10^{6}\right)$ stably expressing Jab1 were subcutaneously injected into the right hind legs of 4-week-old female specific pathogen-free Balb/c nude mice (Beijing Huafukang Bioscience, Beijing, China), and control group cells $\left(1 \times 10^{6}\right)$ were inoculated into the left hind legs of nude mice $(n=20) .22$ days later, the athymic mice were sacrificed and photographed, and xenograft tumors were excised and measured. A Vernier caliper was used to measure the tumor diameter. The tumor volume was calculated following the formula: long diameter multiplied by the square of the short diameter, then divided by two. IHC and H\&E analyses were performed on the tumor tissues. The protocols for animal studies were approved by the Institutional Animal Care and Use Committee of Xuzhou Medical University.

\section{Statistical analysis}

Data were analyzed using SPSS 16.0 software. Statistical significance of Student's t-test was presented for two-group comparisons. More than two groups were examined by one-way or two-way analysis of variance. Kaplan-Meier analysis was performed to examine the association of Jab1 expression and survival of GC patients. Univariate and multivariate Cox regression analysis was performed to access the prognostic factors for survival. $P<0.05$ was statistically significant. The correlations between Jab1 expression and clinicopathologic characteristics were analyzed using Pearson's Chi-square test or Fisher's exact test in GC tissues and para-cancer tissues. Differences between groups were statistically significant when $P<0.05$.

\section{Results}

\section{The association between Jab1 expression and clinical features in GC}

To evaluate the Jab1 expression patterns in human GC, we randomly selected 20 GC tissues and adjacent cancer tissues. Protein samples were extracted from these tissues and detected by WB, and the results showed that Jab1 in tumor tissues has significant higher expression than that in paracancer tissues (Fig. 1a, b, $P<0.01$ ). Consistent with the previous studies, we demonstrated that the expression of Jab1 in GC cells is higher than that in normal gastric epithelial cells [24]. We then analyzed Jab1 expression using IHC in the tissue microarray of $90 \mathrm{GC}$ specimens to investigate the clinical implication of Jab1 protein. GC tissues were judged using H\&E staining, and then the immunoreactivity of Jab1 was assessed in the cytoplasm and nucleus of GC cells (Fig. 1c). The Jab1 staining in GC tissues was observably higher than those in para-cancer tissues.

Then we evaluated the relationships between Jab1 expression and clinicopathological features (according to TNM classification) in GC (Table 1). Statistical analysis revealed that increased Jab1 expression significantly correlated with tumor size $(P=0.002)$, pT status $(P=0.002)$, pN status $(P=0.012)$ and tumor stage $(P=0.049)$, However, there were no correlations with sex and age (Table 1$)$. We further investigated the significance of Jab1 expression for the clinical outcome of patients with GC, and Kaplan-Meier curves showed that patients with high expression of Jab1 is associated with reduced OS (Fig. 1d). To further evaluate the role of Jab1 in GC prognosis, the expression and the clinicopathological parameters found to be significant by univariate analysis were included in the multivariate analysis. As shown in table S1, the covariates included in the Cox regression model were sex, age, tumor size, tumor Stage, pT status, pN status and Jab1 expression. The data indicated that Jab1 expression was an independent prognostic factor for patients with GC. The above results showed that Jab1 is involved in carcinogenesis and tumor progression and could be proposed as promising prognosis marker of GC. 
a

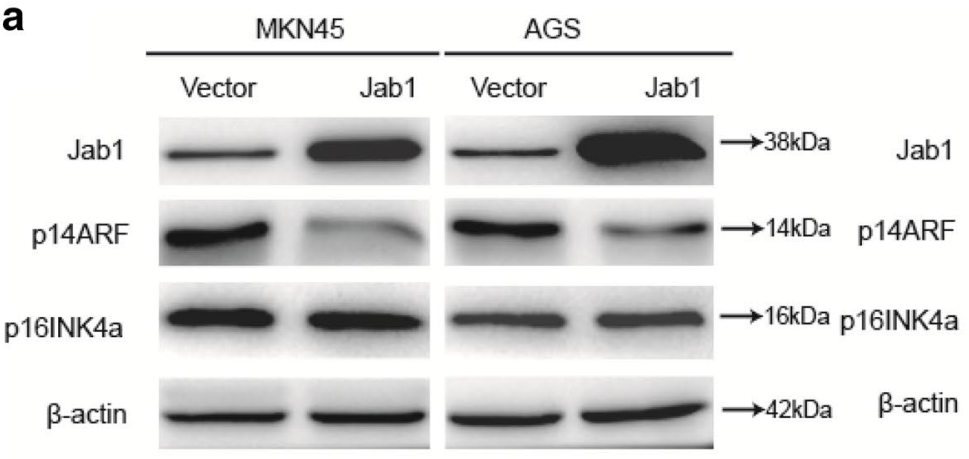

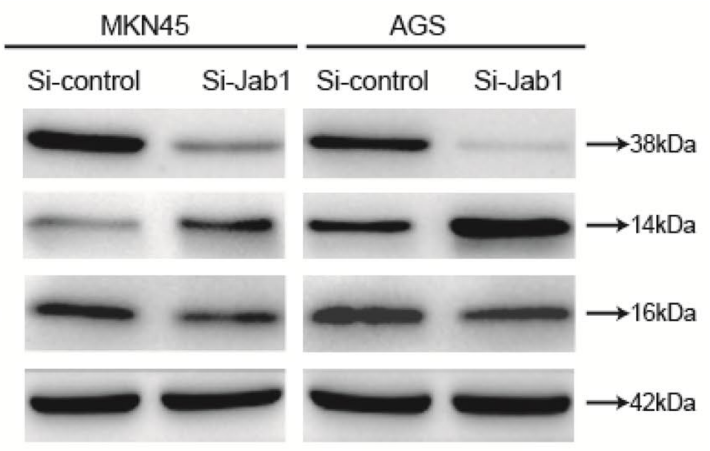

b
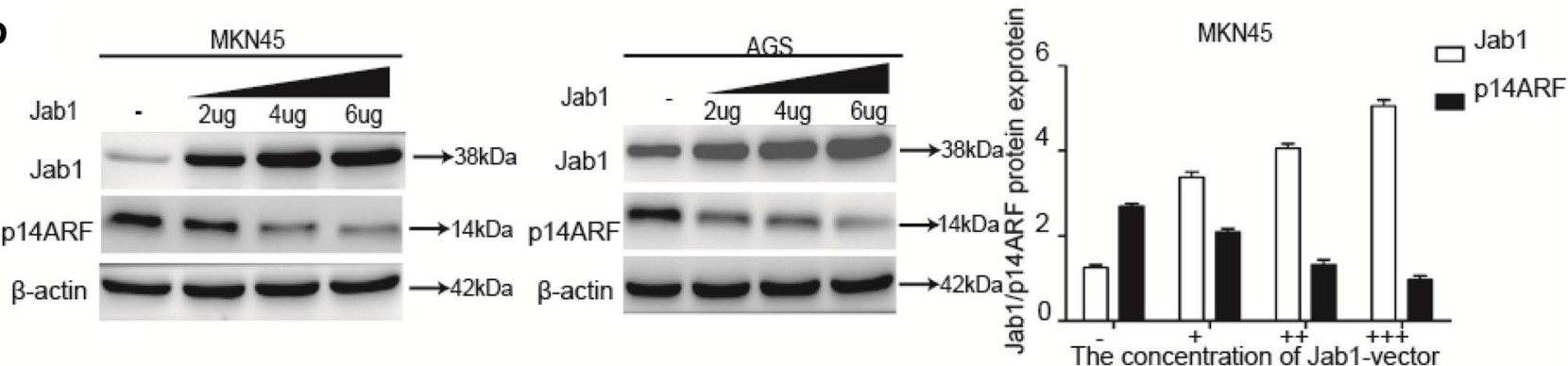

C
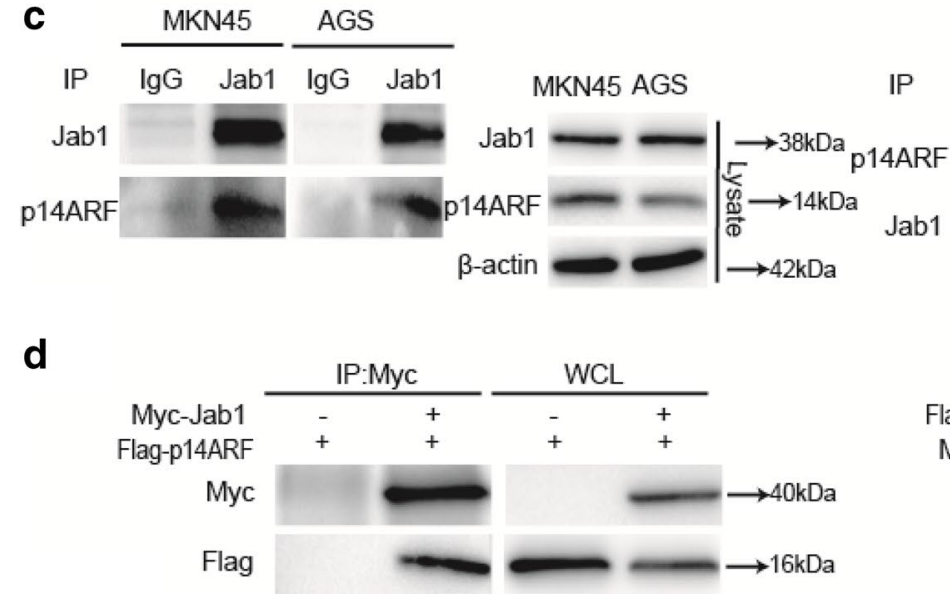
IP $\frac{\text { MKN45 }}{\operatorname{lgG} \text { p14ARF }} \frac{\text { AGS }}{\operatorname{lgG} \text { p14ARF }}$

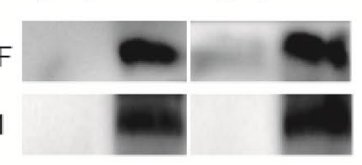

MKN45 AGS

d
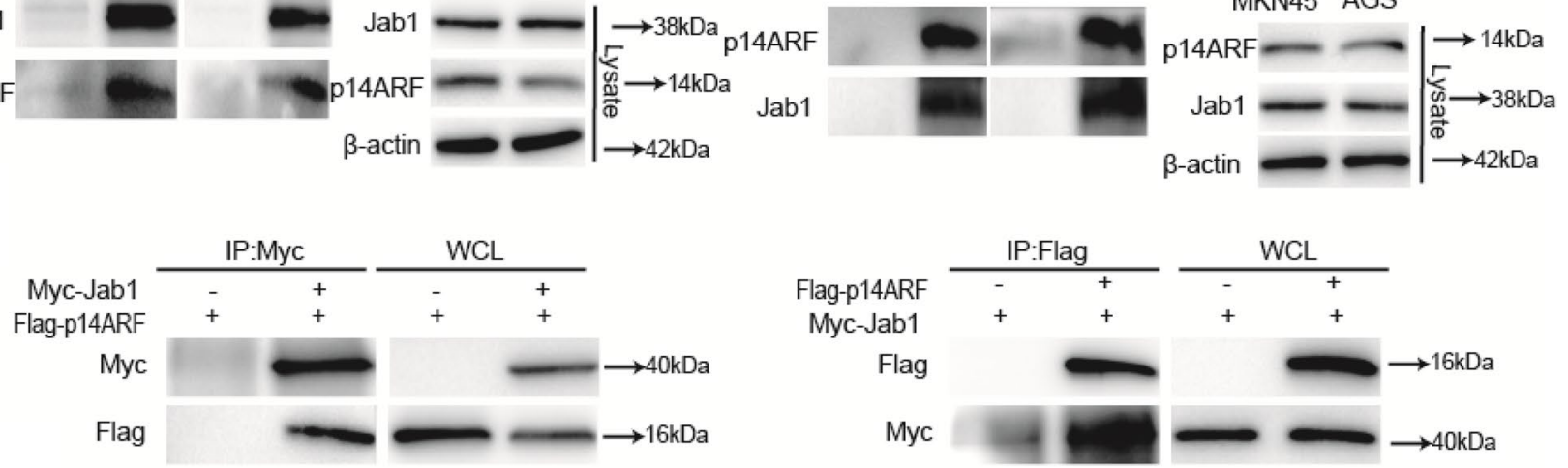

e
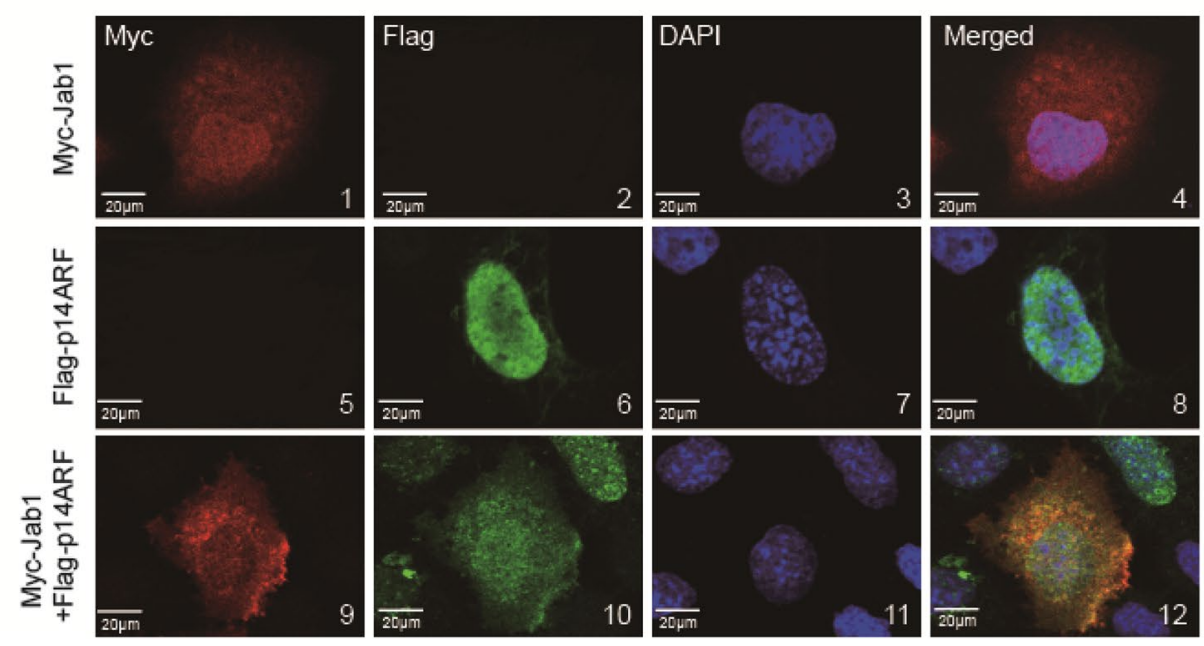
४Fig. 4 Jab1 interacts with p14ARF. a Protein lysates were prepared from Jab1 overexpression or Si-Jab1 transfected cells and immunoblotted with anti-Jab1, anti-p14ARF, and anti-p16INK4a antibody. Jab1 overexpression enhanced the expression of p14ARF. Treatment of Jab1-siRNA (Si-JAB1) induced endogenous p14ARF expression. b Jab1 induces the degradation of p14ARF in two human gastric cancer cell lines in a dose-dependent manner. The cells were transfected with plasmids that expressing Jab1 with increasing concentrations, the protein lysates were prepared from them and detected with anti-Jab1, p14ARF, and $\beta$-actin antibodies. c Endogenous Jab1 and endogenous p14ARF specifically interact with each other. MKN45 and AGS cell lysates were immunoprecipitated (IP) with either an isotype-matched control IgG or the indicated anti-Jab1 antibody and anti-p14ARF antibody. After that, immunoblotted (IB) and lysates (also means input) were determined by WB using the same antibodies. d Co-IP assays was used to confirm the interaction between exogenous Jab1 (Myc-Jab1) and exogenous p14ARF (Flag-p14ARF) in gastric cancer cells. MKN45 cells were transfected with plasmids encoding Myc-Jab1 or with Flag-p14ARF (the opposite works), and whole cell lysates (WCL) were subjected to IP as indicated and IB analysis. e MKN45 cells were transfected with plasmids expressing Myc-Jab1 or Flag-p14ARF separately. Meanwhile, the two plasmids mentioned above were co-transfected into the cells. $24 \mathrm{~h}$ later, fixed cells were detected by anti-Myc or anti-Flag antibodies and stained by Alexa Fluor 488-conjugated antibodies and TRITC that matched the species of Myc and Flag antibodies, evaluated by using N-SIM super-resolution microscopy $(\times 150$ magnification $)$

\section{Jab1 promotes the cell proliferation and cell cycle progression of GC cells in vitro}

Given the association between Jab1 expression and proliferative and aggressive phenotype in GC, we next investigated the effects of ectopic expression of Jab1 on biological characteristics in GC cells. Protein levels of Jab1 markedly up-regulated in MKN45 and AGS cells transfected with Jab1 plasmid compared with non-transfected cells (Fig. 2a). We found that the proliferative ability of GC cells increased after Jab1 overexpression by CCK- 8 assay (Fig. 2b). Enforced expression of Jab1 potentiated the colony forming capacity of MKN45 and AGS cells (Fig. 2c). Moreover, alteration of cell cycle and apoptosis rate was quantified using flow cytometry in GC cells expressing Jab1. Elevate expression of Jab1 accelerated cell cycle progress in MKN45 and AGS cells, which contributed to the decrease in $\mathrm{G} 1$ phase and increase in $\mathrm{S}$ phase (Fig. 2d). Also, apoptosis rate of GC cells treated with exogenous Jab1 decreased (Fig. 2e).

Inversely, we used siRNA targeting Jab1 gene to attenuate Jab1 expression of GC cells to determine the effects of Jab1 knockdown on cell survival and growth. Protein levels of Jab1 markedly down-regulated in MKN45 and AGS cells when transfected with Jab1-siRNA (Fig. 3a). Silencing endogenous Jab1 significantly decreased cellular proliferation and colony formation (Fig. 3b, c). Knockdown of Jab1 showed cell cycle retardation in both cells, as the percentage of cells in G1 stage increased (Fig. 3d).
Furthermore, Jab1 depletion initiated a higher apoptotic rate in comparison with control (Fig. 3e). Taken together, these results revealed that Jab1 potentiates the cellular growth and survival ability of GC cells.

\section{Jab1 interacts with p14ARF and decrease its expression in cells}

In vitro experiments confirmed that Jab1 affects cell proliferation and cell cycle progression, we further explored molecular mechanisms of Jab1-mediated cell activity in GC. Then we found that the expression of p14ARF had an inverse relationship of Jab1 in MKN45 and AGS cells. However, there was no significant alternation in p16INK4a expression (Fig. 4a), which provided a feasible direction for our next studies. p14ARF characterized as tumor-suppressor gene has been reported to be implicated in the regulation of cell cycle, programmed cell death, carcinogenesis and development of tumor $[4,6]$. Therefore, we hypothesize that Jab1 acts on p14ARF, regulating the progression of gastric carcinogenesis.

To further affirm that Jab1 negatively regulating p14ARF is an inevitable consequence rather than a coincidental happening, we gradually increased the concentration of exogenous Jab1 plasmid transfected into MKN45 and AGS cells and then measured the levels of p14ARF protein. Results from western blot revealed that Jab1 amplification resulted in a prominent dose-dependent decrease of p14ARF expression in GC cells (Fig. 4b). Reciprocal Co-IP assays were employed to identify the physical interaction between Jab1 and p14ARF. As presented in Fig. 4c, Jab1 and p14ARF antibody rather than control $\mathrm{IgG}$ precipitated endogenous p14ARF and Jab1 proteins in MKN45 and AGS cells, separately and efficiently. Furthermore, exogenous Myc-Jab1 and Flag-p14ARF plasmids were co-transfected into two cells. Co-IP analysis was performed with anti-Myc and antiFlag antibodies, and the physical interaction between Flagp14ARF and Myc-Jab1 proteins was confirmed (Fig. 4c, d). These interactions drove us to observe intracellular localization of Jab1 and p14ARF in GC cells. Next, the distribution patterns of Jab1 and p14ARF proteins in cells were revealed by immunofluorescence staining and confocal microscopy. Consistent with the previous studies [27, 28], when Jab1 or p14ARF plasmids were transfected independently in MKN45 cells, Jab1 expressed in both the cytoplasm and nucleus and p14ARF predominantly located in the nucleus. Unexpectedly, overlapping fluorescence signals between Jab1 and p14ARF were detected in co-transfected MKN45 cells that p14ARF translocation from nucleus to cytoplasm (Fig. 4e), which confirmed the subcellular co-localization of the two proteins. 
a

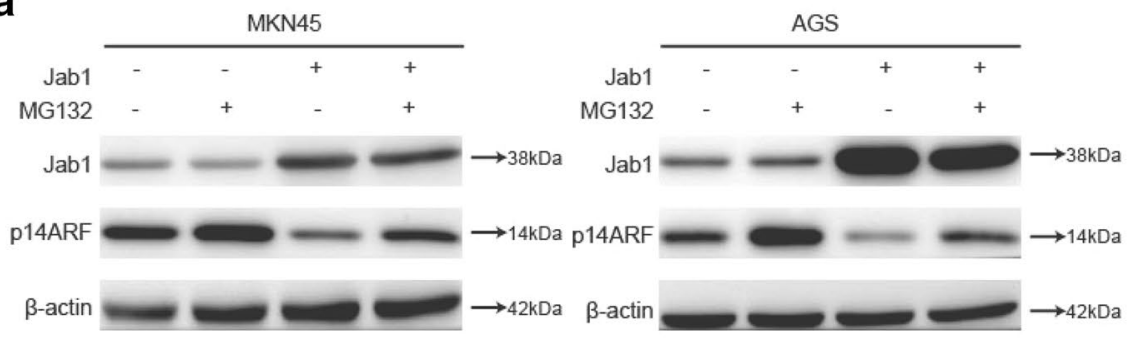

b
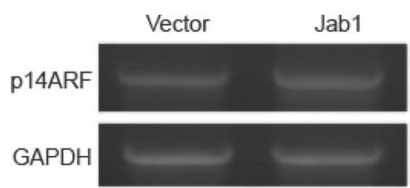

C
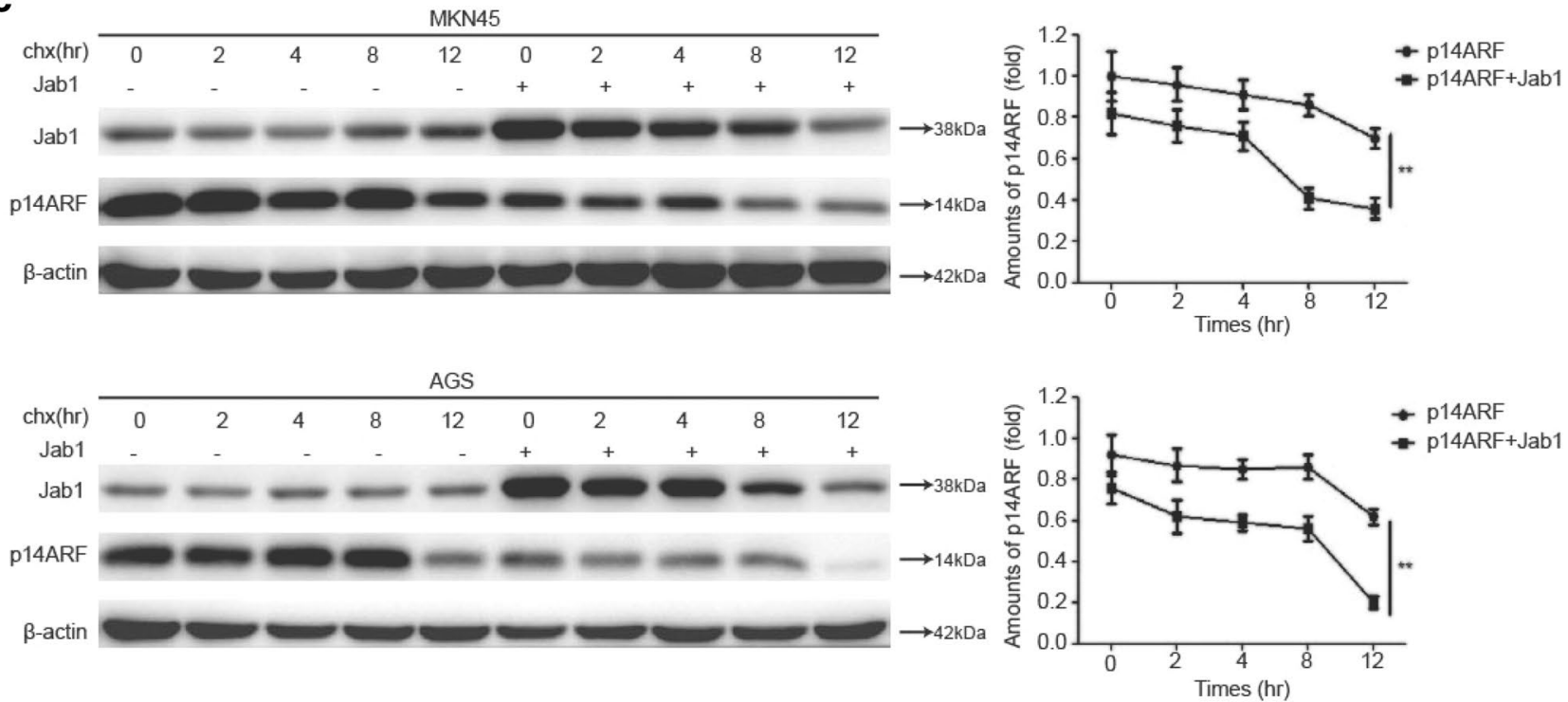

d

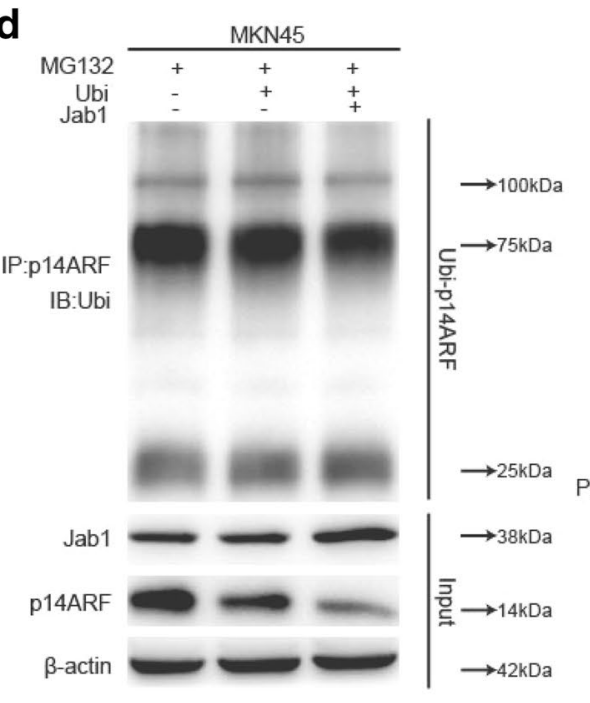

e

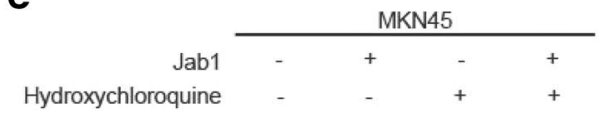

Jab1 $\longrightarrow \rightarrow 38 \mathrm{kDa}$

p14ARF $-\longrightarrow 14 \mathrm{kDa}$

$\beta$-actin $\longrightarrow$

MKN45
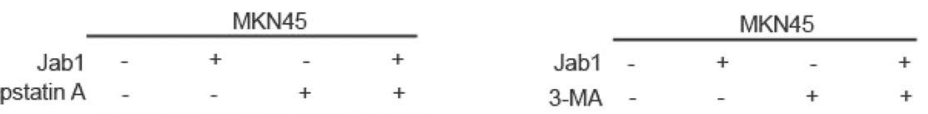

Jab1

$\longrightarrow \rightarrow 38 \mathrm{kDa}$

Jab1 $\longrightarrow \longrightarrow 38 \mathrm{kDa}$

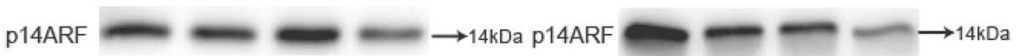

$\beta$-actin $\longrightarrow \longrightarrow 42 \mathrm{kDa} \beta$-actin $\longrightarrow$

$\mathbf{f}$

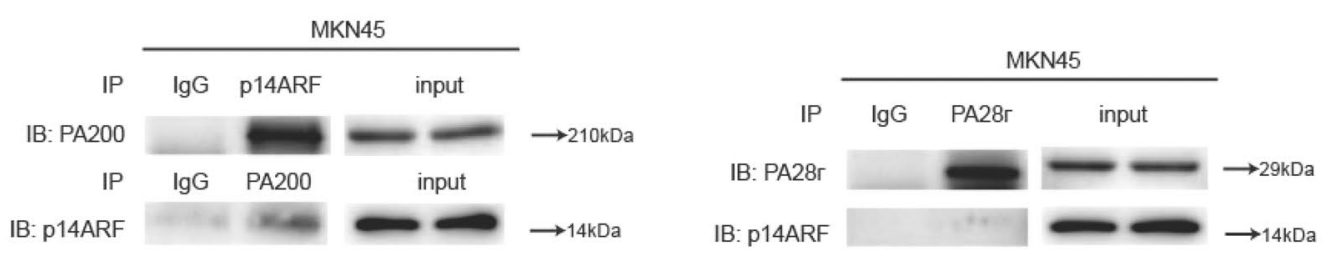


४Fig. 5 Jab1 negatively regulates p14ARF stability in gastric cancer cells. a Jab1 induces proteasomal degradation of p14ARF (the Jab1mediated degradation of p14ARF is proteasome-dependent). MKN45 and AGS cells were transfected with plasmids expressing Jab1 (Jab1 vector) or pcDNA3.1 (a hollow vector, works as control group), and subsequently treated with $20 \mu \mathrm{M}$ MG132 for $6 \mathrm{~h}$ before harvest, the WCL were immunoblotted with Jab1 and p14ARF antibodies. $\beta$-actin was used as control. b The mRNA levels of p14ARF were checked when MKN45 cells were transfected with plasmids expressing Jab1 or pcDNA3.1. c Exogenous Jab1 reduces p14ARF stability and turnover rate. Two human gastric cancer cells transfected with the indicated plasmids were exposed to cycloheximide (CHX) and collected at the indicated time, and subsequently assessed by WB. (The graphs signify the relative amounts of p14ARF compared with the level of $\beta$-actin expression in the WB. The protein level was determined by the Image J program). d The MKN45 cells were co-transfected with the indicated plasmids. The whole cell protein was detected by Co-IP with anti-p14ARF and then IB with anti-ubiquitin. Jab1 has no effect on the ubiquitination of p14ARF protein. e MKN45 cells were transfected with plasmids expressing Jab1 (Jab1 vector) or pcDNA3.1 (a hollow vector, works as control group), and subsequently treated with hydroxychloroquine, 3-MA and pepstatin A before harvest, the WCL was immunoblotted with Jab1 and p14ARF antibodies. $\beta$-actin was used as control. $\mathbf{f}$ The AGS cell lysates were immunoprecipitated using anti-p14ARF, anti-PA200, and anti-PA28г antibodies

\section{Jab1 mediates p14ARF degradation without ubiquitylation modification}

Jab1 has emerged as a regulator of E3 ubiquitin ligase to affect activity and stability of substrate proteins [29], combined with above observations, which urge us to further test and verify whether down-regulation of p14ARF by Jab1 is mediated through the proteasome pathway. In cell experiments, results showed that p14ARF expression elevated in MKN45 and AGS cells treated with proteasome inhibitor MG132, compared to the control group treated with dimethyl sulfoxide (Fig. 5a). In addition, MG132 overrode and blocked Jab1-mediated depletion of p14ARF (Fig. 5a), implying that Jab1 effectuated p14ARF degradation in GC cells via proteasome-dependent manner.

To address whether Jab1 modulated p14ARF turnover on the posttranslational level, we checked the mRNA levels of p14Arf after overexpression of Jab1. Results showed no change of mRNA levels of p14Arf (Fig. 5b). Then we administered MKN45 and AGS cells with protein synthesis inhibitor $\mathrm{CHX}$ at corresponding interval to evaluated the effect of Jab1 on stability of protein p14ARF. Protein stability in GC cells expressing Jab1 decreased by $70 \%$ to $80 \%$ after CHX treatment (Fig. 5c), we concluded that Jab1 acts as an inhibitor of p14ARF through decreasing its stabilization.

Subsequently, we speculated that Jab1 could promote the ubiquitination of p14ARF. Inconsistent with the inference, we detected no alternation in level of p14ARF ubiquitination in GC cells when Jab1 was up-regulation or not (Fig. 5d, Fig. S1A). Because ubiquitination pathway could not decipher
Jab1-mediated p14ARF degradation, the possibility of autophagy related pathway or lysosome dependent manner was further examined in GC cells. The autophagy inhibitors Hydroxychloroquine and 3-MA could not rescue p14ARF degradation excluding involvement of other autophagy processes (Fig. 5e, Fig. S1B). Furthermore, no additional stabilization of p14ARF was observed when treated with the lysosomal inhibitors Pepstatin A (Fig. 5e, Fig. S1B). After adjusting the experimental scheme, we introduced PA200 and PA28 5 to verify the pathway of p14ARF degradation. The observations suggest that endogenous PA200 was pulled down by anti-p14ARF antibody, and endogenous p14ARF was also pulled down by anti-PA200 antibody in GC cells (Fig. 5f, Fig. S1C). It is necessary to add that there is no binding relationship between $\mathrm{p} 14 \mathrm{ARF}$ and PA28г proteins (Fig. 5f, Fig. S1C). To sum up, these results indicated that Jab1 mediates p14ARF via non-ubiquitin proteasome pathway, which possibly requires the PA200 involvement.

\section{Jab1 promotes tumorigenicity in vivo through down-regulating p14ARF expression}

To further identify the role of Jab1 in vivo GC tumorigenesis, we generated MKN45 cells with lentivirus encoding Jab1 to achieve stable Jab1 expression. Stable transfected MKN45 cells were subcutaneously injected into the hind legs of nude mice (left leg: cells containing control lentivirus; right leg: cells containing Jab1 lentivirus) to establish xenograft models. Xenograft tumors were excised from the nude mice after twenty-two days (Fig. 6a). As anticipated, the difference of xenograft tumor was palpable, and tumor weights and volumes in Jab1 overexpression group were significantly greater than those in the control group (Fig. 6b, c). IHC staining was performed on the histopathologic slides of tumor tissues excised from nude mice, and the results verified inverse relationship between Jab1 and p14ARF levels (Fig. 6d). Moreover, we examined pathological sections of $10 \mathrm{GC}$ specimens to compare IHC staining of tumor with para-non-tumor tissue. We found that the levels of Jab1 protein were increased and the levels of p14ARF were decreased in the tumor tissue compared to levels in the non-tumor tissue (Fig. 6e). In brief, upregulation of Jab1 promotes GC growth in vivo by targeting p14ARF.

\section{Discussion}

Jab1 was initially reported to be a transcriptional coactivator of c-Jun, and subsequently discovered as the fifth unit of COP9 signalosome complex. Accumulating evidence suggests that Jab1 is implicated in modulating the biological functions of tumor cells, including carcinogenesis and tumor progression [20], DNA damage and repair [30], cell 
a

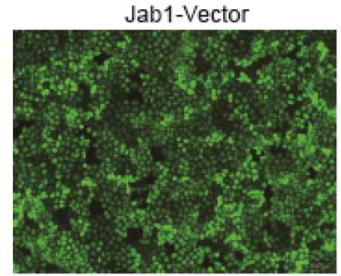

Left: Jab1-Vector

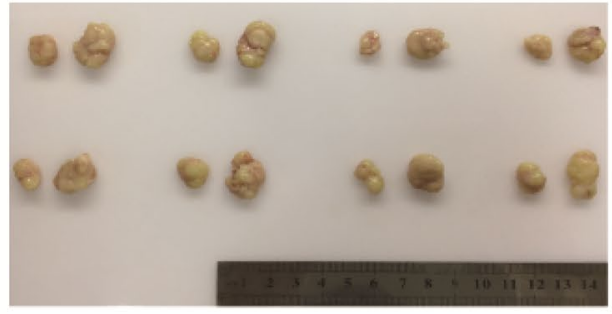

d

H\&E

Jab1
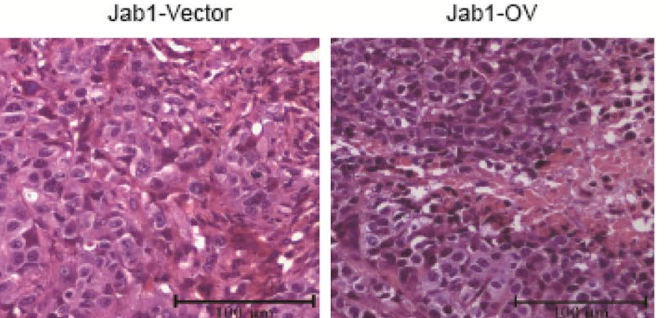

p14ARF
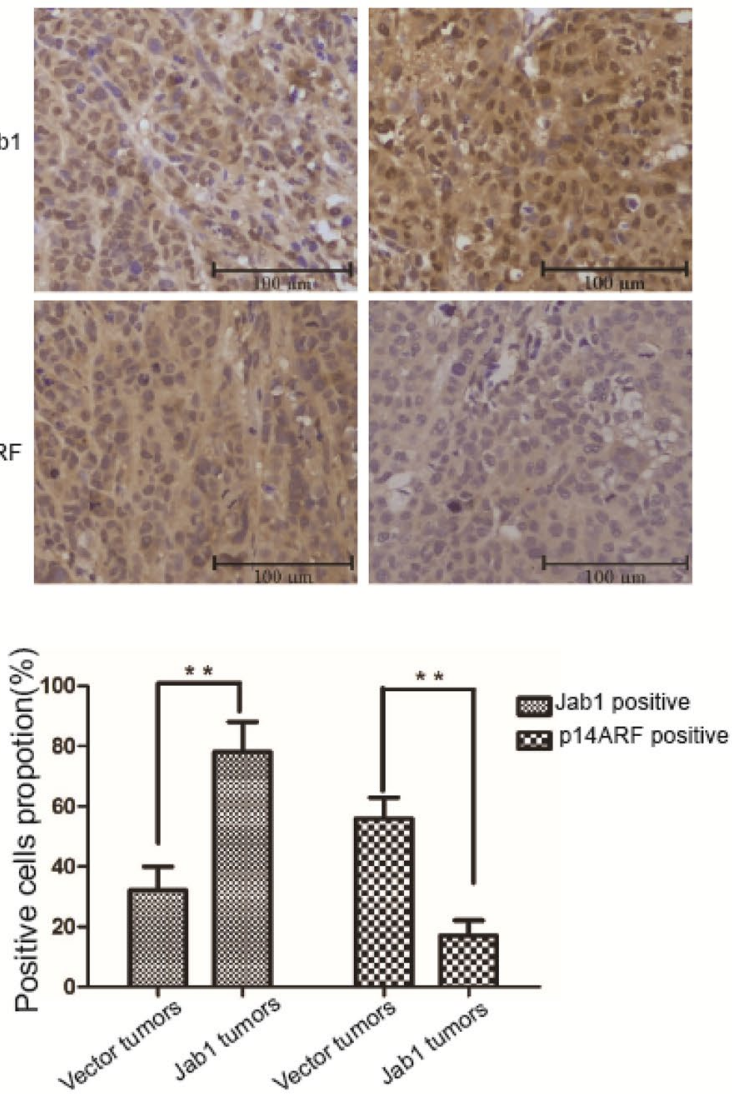
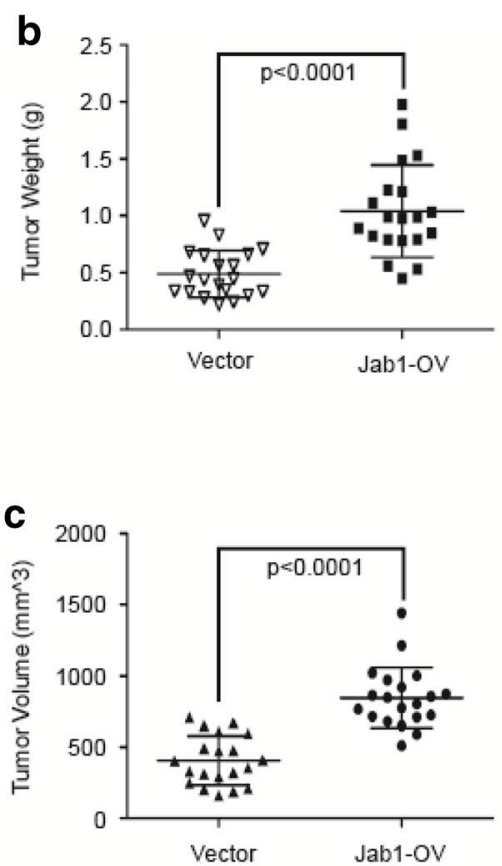

e Paratumor Tumor
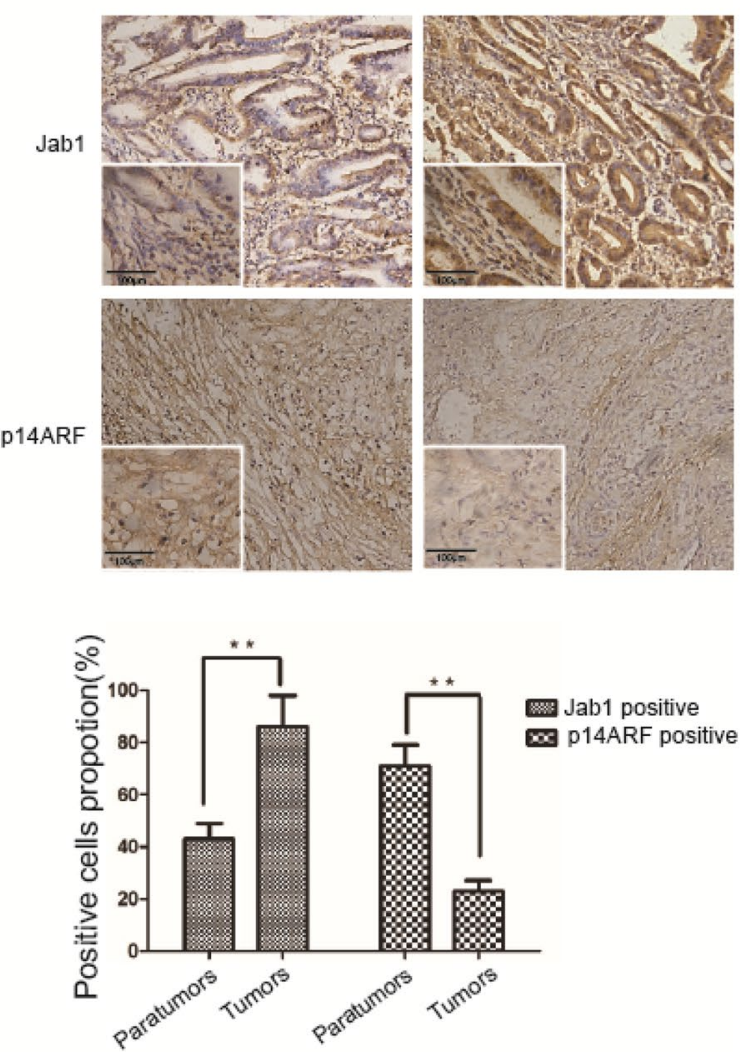
4Fig. 6 High expression of Jab1 accelerated the growth of gastric cancer cells in vivo. a $1 \times 10^{6}$ MKN45 cells transfected with lentiviruses which could stably express Jab1 were injected into the nude mice right hind leg, and the control group cells were injected into mice left hind leg $(n=20)$. At 22 days after injection, the mice were sacrificed. The tumors from mice were photographed. b Tumor weights were displayed as means \pm SD from 20 mice in each group. $c$ The quantification of tumor volumes excised from 20 mice in two groups was shown. d The levels of Jab1 and p14ARF expression showed the inverse relationship in mice tumor tissues, original magnification $\times 400$. e The levels of Jab1 and p14ARF protein were examined in gastric cancer tissues and adjacent non-tumor tissues in $10 \mathrm{GC}$ specimens by IHC staining, original magnification $\times 200$, insert magnification $\times 400$

proliferation and apoptosis [24], and multiple signal transduction pathways [20]. Ectopic expression and amplification of Jab1 has been proved in multiple aggressive malignancies, such as breast cancer, hepatocellular carcinoma, and pancreatic cancer, and others [31-33], supporting the role of Jab1 as an oncogene. However, detailed molecular mechanisms of Jab1 in GC remain unclear. In our study, we found that Jab1 may function as a suppressor of p14ARF to facilitate the formation and development of GC.

In this study, we explored the Jab1 levels in GC and paracancer tissues, and the protein level of Jab1 was enhanced in cancer in comparison with para-cancer. Jab1 overexpression had proliferative phenotypes and unfavorable prognosis of GC patients. Our in vitro experiment exhibited reciprocal effects of up-regulation and down-regulation of Jab1 on malignant biological functions in GC cells, supporting the pro-oncogenesis role of Jab1. Enforced expression Jab1 potentiated proliferation, accelerated cell cycle progress, and inhibited apoptosis of GC cells. Conversely, interfering Jab1 expression in GC cells may reduce cellular proliferation, retard cell cycle process, and expedite cell apoptosis. Besides, our results showed that Jab1 negatively regulates p14ARF expression in GC cells. As a tumor suppressor gene, p14ARF has received extensive attention in the recent years $[12,34]$. The critical regulatory mechanisms of Jab1 influencing p14ARF expression were emphasized by the observations that Jab1 could destabilize p14ARF protein and promote its degradation, which requires the involvement of PA200 in the process of non-ubiquitination proteasome hydrolysis.

Ubiquitination, an extremely major protein posttranslational modification, regulates the location, metabolism and degradation of substrate proteins and biological activities of cells, in the process of which ubiquitin ligases E3 responsible for specific recognition of target proteins acts a decisive role. Previous studies have repeatedly confirmed that Jab1 could act as a regulator of E3 ubiquitin ligase involved in the degradation of target proteins via ubiquitin-proteasome pathway [29]. However, after examining the amino acid composition of p14ARF protein, we found that the amino acids that make up p14ARF polypeptide chains do not contain lysine covalently linking to ubiquitin in ubiquitination modification, which means the degradation of p14ARF could not be mediated by ubiquitination pathway. However, its decreasing tendency can be blocked by proteasome inhibitor MG132. In other words, the degradation of p14ARF is through the non-ubiquitin-proteasome pathway induced by Jab1.

Proteasome primarily degrades the dysfunctional or damaged proteins through hydrolyzing its peptide bond. Although most of the proteasome substrates must be ubiquitination prior to degradation, there are still some exceptions, notably in the process of proteasome involvement in the post-translation of proteins. The $26 \mathrm{~S}$ proteasome composed of 20S particle and 19S-regulatory particle is the main molecular machine responsible for protein degradation in eukaryotic cells [35]. At least four proteins that activate 20S core particles have been found: PA28, PA200, 19 s regulatory particles and P97. Several studies have shown that PA28 and PA200 activate 20S core particle degradation proteins without hydrolyzing ATP to provide energy, unable to recognize and degrade ubiquitin chain-labeled protein substrate $[36,37]$. PA200 protein has been previously documented to act as a proteasome activator by interacting with $20 \mathrm{~S}$ particle [22]. To the best of our knowledge, ornithine decarboxylase (ODC) [38], Rb protein [39], c-Fos protein [40], and even the familiar p53 protein [41] are degraded by non-ubiquitin proteasome pathway. Our findings confirmed that p14ARF protein was degraded by a non-ubiquitin proteasome pathway after binding to PA200 and Jab1.

In order to verify the possible relationship between Jab1 and p16-INK4a, we performed western blot and found no corresponding regulating effects between Jab1 and p16-INK4a. Thus, Jab1 regulated p14ARF, but not p16-INK4a, suggesting that the processes by which Jab1 regulates the two proteins encoded by CDKN2A are independent of each other [7, 8]. The specific mechanistic effects of Jab1 on the promoter for encoding the p14ARF protein require further study. Regarding the degradation of p14ARF induced by Jab1, immunofluorescent assay showed that the vast majority of p14ARF protein over-expressed in gastric cancer cells were localized in the nucleus, whereas the co-expression of Jab1 led to the reorientation of p14ARF to the cytoplasm. In the study of p14ARF protein translocation, previous researchers have shown that p14ARF seemed to be more likely to form complexes with other proteins, and that, under specific factors, the complex was transferred into the nucleus [12, 42]. After consulting relevant literatures, we found that the study of specific mechanism about p14ARF nuclear export was scarce. Previous studies have confirmed that p27 harboring/containing NES sequence shuttles between cytoplasm and nucleus, which can be regulated by the combination of Jab1 in a CRM1-dependent manner [20, 43]. By analogy, we wonder whether Jab1 can 
specifically bind to a certain site of p14ARF protein and promote the nuclear export of p14ARF with the coordination of specific proteins.

Taken together, we draw noteworthy conclusions that high expression of Jab1 could induce the degradation of the p14ARF protein, and then contributing to the increased growth potential of GC cells. In consequence, Jab1 is a potential novel crucial therapeutic target for the treatment of GC. In addition, a comprehensive understanding of the protein degradation process by non-ubiquitin dependent proteasome will provide theoretical guidance for the development and utilization of specific drugs, and provide a new breakthrough for the treatment of cancer.

Acknowledgements This work was supported by the National Natural Science Foundation of China (Nos. 81872080, 81572349), Jiangsu Provincial Medical Talent (ZDRCA2016055), the Science and Technology Department of Jiangsu Province (BK20181148), the Priority Academic Program Development of Jiangsu Higher Education Institutions (PAPD) and the 333 high-level talents of Jiangsu Province (BRA2019083).

Author contributions LW, WQD and DSP designed, performed and analyzed experiments. LW and WQD performed experiments. LW, MX and MRL wrote the paper. FCH and JY commented on the study and revised the paper. DSP obtained funding and designed the research.

\section{Compliance with ethical standards}

Conflict of interest The authors declare no competing interests.

Ethics approval and consent to participate Patient tissue samples were obtained with informed consent, under the protocol approved by the ethical review board of the Affiliated Hospital of Xuzhou Medical University.

Consent for publication The authors confirm that they have obtained written consent from each patient to publish the manuscript.

Availability of data and materials All data generated or analyzed during this study are included in this article.

\section{References}

1. Casamayor M, Morlock R, Maeda H, Ajani J. Targeted literature review of the global burden of gastric cancer. Ecancermedicalscience. 2018;12:883.

2. Baniak N, Senger JL, Ahmed S, Kanthan SC, Kanthan R. Gastric biomarkers: a global review. World J Surg Oncol. 2016;14:212.

3. Carlomagno N, Incollingo P, Tammaro V, Peluso G, Rupealta N, Chiacchio G, et al. Diagnostic, predictive, prognostic, and therapeutic molecular biomarkers in third millennium: a breakthrough in gastric cancer. Biomed Res Int. 2017;2017:7869802.

4. Inoue K, Fry EA. Aberrant expression of p14(ARF) in human cancers: a new biomarker? Tumor Microenviron. 2018;1:37-44.

5. 5Fontana R, Vivo M. Dynamics of p14ARF and focal adhesion kinase-mediated autophagy in cancer. Cancers 2018;10.

6. Luchinat E, Chiarella S, Franceschini M, Di Matteo A, Brunori M, Banci L, et al. Identification of a novel nucleophosmin-interaction motif in the tumor suppressor p14arf. FEBS J. 2018;285:832-47.
7. Quelle DE, Zindy F, Ashmun RA, Sherr CJ. Alternative reading frames of the INK4a tumor suppressor gene encode two unrelated proteins capable of inducing cell cycle arrest. Cell. 1995;83:993-1000.

8. Gibson SL, Dai CY, Lee HW, DePinho RA, Gee MS, Lee WM, et al. Inhibition of colon tumor progression and angiogenesis by the Ink4a/Arf locus. Can Res. 2003;63:742-6.

9. Stone S, Jiang P, Dayananth P, Tavtigian SV, Katcher H, Parry D, et al. Complex structure and regulation of the P16 (MTS1) locus. Can Res. 1995;55:2988-94.

10. Silva J, Silva JM, Dominguez G, Garcia JM, Cantos B, Rodriguez $\mathrm{R}$, et al. Concomitant expression of p16INK4a and p14ARF in primary breast cancer and analysis of inactivation mechanisms. $\mathrm{J}$ Pathol. 2003;199:289-97.

11. Nicholson SA, Okby NT, Khan MA, Welsh JA, McMenamin MG, Travis WD, et al. Alterations of p14ARF, p53, and p73 genes involved in the E2F-1-mediated apoptotic pathways in non-small cell lung carcinoma. Can Res. 2001;61:5636-43.

12. Cai JB, Shi GM, Dong ZR, Ke AW, Ma HH, Gao Q, et al. Ubiquitin-specific protease 7 accelerates p14(ARF) degradation by deubiquitinating thyroid hormone receptor-interacting protein 12 and promotes hepatocellular carcinoma progression. Hepatology. 2015;61:1603-14.

13. Chamovitz DA, Segal D. JAB1/CSN5 and the COP9 signalosome. A complex situation. EMBO Rep. 2001;2:96-101.

14. Chamovitz DA, Wei N, Osterlund MT, von Arnim AG, Staub JM, Matsui M, et al. The COP9 complex, a novel multisubunit nuclear regulator involved in light control of a plant developmental switch. Cell. 1996;86:115-21.

15. Claret FX, Hibi M, Dhut S, Toda T, Karin M. A new group of conserved coactivators that increase the specificity of AP-1 transcription factors. Nature. 1996;383:453-7.

16. Wei N, Chamovitz DA, Deng XW. Arabidopsis COP9 is a component of a novel signaling complex mediating light control of development. Cell. 1994;78:117-24.

17. Guo H, Jing L, Cheng Y, Atsaves V, Lv Y, Wu T, et al. Downregulation of the cyclin-dependent kinase inhibitor p57 is mediated by Jab1/Csn5 in hepatocarcinogenesis. Hepatology. 2016;63:898-913.

18. Tomoda K, Kubota Y, Kato J. Degradation of the cyclin-dependent-kinase inhibitor p27Kip1 is instigated by Jab1. Nature. 1999;398:160-5.

19. Bae MK, Ahn MY, Jeong JW, Bae MH, Lee YM, Bae SK, et al. Jab1 interacts directly with HIF-1alpha and regulates its stability. J Biol Chem. 2002;277:9-12.

20. Wang L, Zheng JN, Pei DS. The emerging roles of Jab1/CSN5 in cancer. Med Oncol. 2016;33:90.

21. Pan Y, Yang H, Claret FX. Emerging roles of Jab1/CSN5 in DNA damage response, DNA repair, and cancer. Cancer Biol Ther. 2014;15:256-62.

22. Blickwedehl J, Olejniczak S, Cummings R, Sarvaiya N, Mantilla A, Chanan-Khan A, et al. The proteasome activator PA200 regulates tumor cell responsiveness to glutamine and resistance to ionizing radiation. Mol Cancer Res. 2012;10:937-44.

23. Blickwedehl J, Agarwal M, Seong C, Pandita RK, Melendy T, Sung P, et al. Role for proteasome activator PA200 and postglutamyl proteasome activity in genomic stability. Proc Natl Acad Sci USA. 2008;105:16165-700.

24. Sang MM, Du WQ, Zhang RY, Zheng JN, Pei DS. Suppression of CSN5 promotes the apoptosis of gastric cancer cells through regulating p53-related apoptotic pathways. Bioorg Med Chem Lett. 2015;25:2897-901.

25. Huang XY, Ke AW, Shi GM, Zhang X, Zhang C, Shi YH, et al. alphaB-crystallin complexes with 14-3-3zeta to induce epithelialmesenchymal transition and resistance to sorafenib in hepatocellular carcinoma. Hepatology. 2013;57:2235-47. 
26. Ko A, Lee EW, Yeh JY, Yang MR, Oh W, Moon JS, et al. MKRN1 induces degradation of West Nile virus capsid protein by functioning as an E3 ligase. J Virol. 2010;84:426-36.

27. Wei N, Serino G, Deng XW. The COP9 signalosome: more than a protease. Trends Biochem Sci. 2008;33:592-600.

28. Wei N, Deng XW. The COP9 signalosome. Annu Rev Cell Dev Biol. 2003;19:261-86.

29. Cope GA, Deshaies RJ. COP9 signalosome: a multifunctional regulator of SCF and other cullin-based ubiquitin ligases. Cell. 2003;114:663-71.

30. Pan Y, Zhang Q, Atsaves V, Yang H, Claret FX. Suppression of Jab1/CSN5 induces radio- and chemo-sensitivity in nasopharyngeal carcinoma through changes to the DNA damage and repair pathways. Oncogene. 2013;32:2756-66.

31. Kouvaraki MA, Rassidakis GZ, Tian L, Kumar R, Kittas C, Claret FX. Jun activation domain-binding protein 1 expression in breast cancer inversely correlates with the cell cycle inhibitor p27(Kip1). Can Res. 2003;63:2977-81.

32. Hsu MC, Huang CC, Chang HC, Hu TH, Hung WC. Overexpression of Jab1 in hepatocellular carcinoma and its inhibition by peroxisome proliferator-activated receptor $\{$ gamma $\}$ ligands in vitro and in vivo. Clin Cancer Res. 2008;14:4045-52.

33. Kouvaraki MA, Korapati AL, Rassidakis GZ, Tian L, Zhang Q, Chiao $\mathrm{P}$, et al. Potential role of Jun activation domain-binding protein 1 as a negative regulator of p27kip1 in pancreatic adenocarcinoma. Can Res. 2006;66:8581-9.

34. Ko A, Shin JY, Seo J, Lee KD, Lee EW, Lee MS, et al. Acceleration of gastric tumorigenesis through MKRN1-mediated posttranslational regulation of p14ARF. J Natl Cancer Inst. 2012;104:1660-722.
35. Smalle J, Vierstra RD. The ubiquitin $26 \mathrm{~S}$ proteasome proteolytic pathway. Annu Rev Plant Biol. 2004;55:555-90.

36. Stadtmueller BM, Hill CP. Proteasome activators. Mol Cell. 2011;41:8-19.

37. Kish-Trier E, Hill CP. Structural biology of the proteasome. Annual review of biophysics. 2013;42:29-49.

38. Orlowski M, Wilk S. Ubiquitin-independent proteolytic functions of the proteasome. Arch Biochem Biophys. 2003;415:1-5.

39. Kalejta RF, Shenk T. Proteasome-dependent, ubiquitin-independent degradation of the Rb family of tumor suppressors by the human cytomegalovirus pp71 protein. Proc Natl Acad Sci USA. 2003;100:3263-8.

40. Basbous J, Jariel-Encontre I, Gomard T, Bossis G, Piechaczyk M. Ubiquitin-independent- versus ubiquitin-dependent proteasomal degradation of the c-Fos and Fra- 1 transcription factors: is there a unique answer? Biochimie. 2008;90:296-305.

41. Tsvetkov P, Reuven N, Shaul Y. Ubiquitin-independent $\mathrm{p} 53$ proteasomal degradation. Cell Death Differ. 2010;17:103-8.

42. Wang J, Ding S, Duan Z, Xie Q, Zhang T, Zhang X, et al. Role of p14ARF-HDM2-p53 axis in SOX6-mediated tumor suppression. Oncogene. 2016;35:1692-702.

43. Pan Y, Zhang Q, Tian L, Wang X, Fan X, Zhang H, et al. Jab1/ CSN5 negatively regulates $\mathrm{p} 27$ and plays a role in the pathogenesis of nasopharyngeal carcinoma. Can Res. 2012;72:1890-900.

Publisher's Note Springer Nature remains neutral with regard to jurisdictional claims in published maps and institutional affiliations. 